Itinéraires Itinéraires

Littérature, textes, cultures

2010-3 | 2010

Médiévalisme

\title{
Introduction (2). Le château de Malbrouck : un château médiéval d'aujourd'hui
}

Éric Necker

\section{(2) OpenEdition}

\section{Journals}

Édition électronique

URL : http://journals.openedition.org/itineraires/1794

DOI : 10.4000/itineraires. 1794

ISSN : 2427-920X

Éditeur

Pléiade

\section{Édition imprimée}

Date de publication : 1 novembre 2010

Pagination : 27-32

ISBN : 978-2-296-13150-7

ISSN : 2100-1340

\section{Référence électronique}

Éric Necker, «Introduction (2). Le château de Malbrouck : un château médiéval d'aujourd'hui », Itinéraires [En ligne], 2010-3 | 2010, mis en ligne le 01 novembre 2010, consulté le 19 avril 2019. URL : http://journals.openedition.org/itineraires/1794; DOI : 10.4000/itineraires.1794

\section{(C) $(\oplus \Theta$}

Itinéraires est mis à disposition selon les termes de la licence Creative Commons Attribution - Pas d'Utilisation Commerciale - Pas de Modification 4.0 International. 


\title{
Introduction (2). Le château de Malbrouck : un château médiéval d'aujourd'hui
}

\begin{abstract}
Built at the end of the Middle Ages, the castle of Malbrouck, now a property of the Département de la Moselle, was restored between 1991 and 1998. This paper, which is not intended as a historical or archeological study, deals with the image that the castle was supposed to give, at the time of its construction, then of its restoration. The subtle links between (archeological or historical) reality, literature and romantic legends, show how present in our imagination the Middle Ages are.
\end{abstract}

Keywords : architecture, Middle Ages, romanticism, medievalism, Restoration Mots clés : architecture, Moyen Âge, romantisme, médiévalisme, Restauration

Si ce colloque sur le médiévalisme a lieu en ces murs, ce n'est finalement pas par hasard. Un château a son histoire, ses légendes peut-être. Si l'on peut se douter de sa fonction (mais méfions-nous des apparences), si l'on peut le décrire architecturalement parlant, il peut aussi incarner une idée, un homme, un principe. Il est aussi le reflet d'une société aujourd'hui disparue et pourtant si présente.

En quelques mots, je vais essayer de montrer combien ce château dès l'origine a voulu « faire médiéval », alors que le Moyen Âge était à son terme, et que sa restauration récente contribue également à matérialiser une certaine image ou représentation du Moyen Âge. J'évoquerai, presque sous forme de boutade, les relations subtiles entre histoire, légende et architecture ou archéologie qui participent de notre imaginaire médiéval depuis le XIX ${ }^{\mathrm{e}}$ siècle.

\section{Un château fort ou sa représentation?}

Le château de Meinsberg, aujourd'hui château de Malbrouck ${ }^{1}$, a été construit par Arnold VI (1366-1455) de Sierck à partir de 1419. En 1427, la

1. En juin 1705, lors de la guerre de Succession d'Espagne, le duc de Marlborough y résida alors 
construction du château devait être probablement très avancée et en 1436, Arnold VI obtient l'investiture du château par le duc de Lorraine René Ir (1409-1480).

Le château tel qu'il a été conçu présente certes de nombreux éléments défensifs et sa silhouette est encore celle d'un château fort. Cependant, construit à la fin du Moyen Âge, il n'est déjà plus d'une grande efficacité défensive et l'on doit plus parler d'une résidence seigneuriale fortifiée que d'un château fort. L'aménagement des principales tours, tour des Dames et tour de la Lanterne, l'existence d'un spacieux logis seigneurial le montrent d'ailleurs bien. En forçant à peine le trait, nous pourrions affirmer que le château, dès sa construction, était un « anachronisme » architectural dans le sens où la forme architecturale adoptée n'est guère en relation avec l'efficacité défensive qu'elle prétend. Mais pourquoi?

Ce château est d'abord le symbole de la réussite d'une famille noble à l'origine modeste qui a su par des alliances réussies et une fidélité constante aux ducs de Lorraine progresser dans la hiérarchie nobiliaire jusqu'au plus haut niveau. L'allure si médiévale du château, faite de force et de puissance, est d'abord un message adressé aux contemporains d'Arnold VI. Quand la construction du château s'achève, Arnold approche des 70 ans. Le château n'est-il pas alors l'ultime représentation d'une époque révolue que regrette peut-être un homme d'une génération passée? N'est-il pas aussi l'image et le gage d'un certain ordre politique et social idéalisé et fortement symbolique, à un moment où la Renaissance commence à se percevoir chez les élites, comme c'est le cas avec le propre fils d'Arnold, l'archevêque réformateur Jacques III?

\section{Un choix de restauration}

Traversons maintenant les siècles... Le château est classé monument historique en 1930. En 1945, il est bombardé et les fermiers qui habitaient le logis y renoncent lorsque les projets de restauration, au titre des dommages de guerre, sont abandonnés. En 1975, le château en ruine est acquis par le Département qui, dans les années 1970-1980, entreprend des travaux de consolidation de la ruine; différentes études et fouilles archéologiques sont menées durant cette période. La restauration du château commence en 1991 et se terminera en 1998. Entre la ruine des années 1970 et le château tel qu'il apparaît aujourd'hui (voir le cahier photographique), le contraste est évidemment saisissant. Pourquoi avoir mené une telle restauration? À quelle image du Moyen Âge le château nous renvoie t-il aujourd'hui?

Pour ceux qui s'en étonnent, rappelons au préalable que ce château, classé monument historique, a été restauré dans le respect de la Charte

qu'il tentait d'envahir la France par la vallée de la Moselle. Il laissa ainsi son nom au château qui devint le château de Malbrouck, déformation populaire (ou germanique) de son nom. 
internationale sur la conservation et la restauration des monuments et des sites, appelée aussi Charte de Venise, adoptée en 1964. Cette charte demande à l'article 9 que toute restitution ou reconstruction se différencie du bâti historique et porte « la marque de notre temps ». La ruine possédait encore $80 \%$ de ses parements mais il manquait les couronnements des murs et les planchers. C'est bien sûr cette question du traitement des couronnements qui était essentielle. Guy Hauswald, l'ingénieur en chef qui suivit les travaux pour le Département, le souligne :

[...] il fallait donc trouver une expression pour les compléments de maçonneries hautes. Le parti adopté a été de compléter les lacunes de parement par du mœllonnage de faible dimension en opposition au mœllonnage en gros appareil [...] qui exprime suffisamment clairement l'intervention mais ne rompt pas la continuité architecturale de l'édifice. Quant aux couvertures, elles sont restituées en ardoises schuppen de provenance luxembourgeoise, matériaux identiques aux éléments révélés par les fouilles archéologiques ${ }^{2}$.

La restauration n'a donc pas consisté à réaliser un pastiche sur la base d'une structure médiévale ruinée ou pire encore, selon nos critères contemporains tout au moins, de réinventer un nouveau néogothique ou un nouveau revival.

D'autres partis pris auraient pu être choisis : consolider la ruine afin de permettre sa visite avec un espace minimal d'accueil ou intervenir par l'ajout d'éléments d'architecture contemporaine comme au château de Falaise par exemple. Ici, l'architecte des Monuments Historiques a préféré une intervention contemporaine très discrète, permettant de restituer la silhouette médiévale que l'on connaît aujourd'hui. Cette silhouette se détachant au loin frappe l'imagination et la perception que nous en avons quand nous nous approchons reste la même, et cela jusque dans la cour. C'est seulement à l'intérieur que l'on découvre de manière évidente l'intervention contemporaine.

\section{Imaginaire et réalité historique}

Si tous les châteaux écossais ont leur fantôme, le château de Malbrouck a sa légende, son four d'alchimiste et même ses sorcières! Mais où se situent la réalité historique et la légende? Cette dernière est rapportée par la revue savante L'Austrasie, en $1839^{3}$, sur les dires d'Emmanuel d'Huart, érudit bien connu à l'époque. Arnold, présenté comme un Templier, a dissipé sa fortune dans les plaisirs et la débauche et n'a plus les moyens d'achever la construction de son château. Il fait donc appel aux puissances des ténèbres et le diable lui apporte or et longévité en échange de son âme. Lors d'un

2. «Le château de Malbrouck à Manderen », Le Pays Lorrain, juillet-septembre 1998.

3. "Le château de Mensberg », L'Austrasie, janvier 1839, p. 20-21. 
dernier banquet, le diable lui rappelle le pacte qu'il avait évidement oublié : Lucifer, déployant « des ailes de chauve-souris, emporte sa proie à travers les airs $»$ !

L'article a paru en pleine période romantique, dont on connaît le goût pour les ruines. Certains thèmes, chers aux romantiques, se retrouvent de manière diffuse dans le récit; l'image romantique du Templier s'adonnant aux plaisirs et à la débauche transparaît également. Souvent l'imaginaire romantique associait Templiers et alchimie, la pratique de celle-ci pouvant expliquer le mystère de leur richesse !

Du diable à l'alchimie, il n'y a qu'un pas. Arnold VI aurait-il été alchimiste? Il se trouve que les fouilles archéologiques révélèrent la présence d'un grand four dans le sous-sol de la tour de la Lanterne, la tour résidentielle la plus prestigieuse, habitée par le fils d'Arnold VI, le puissant archevêque de Trèves, Jacques III.

Compte tenu de l'originalité de ce four, plusieurs hypothèses ont été émises. L'analyse des restes et des résidus a montré qu'il ne s'agit pas d'un four de fondeur ou de celui d'un atelier monétaire. En revanche, la structure du four du château fait incontestablement penser aux fours de distillation dont on trouve des représentations dans différents ouvrages. La distillation n'est pas une opération à proprement parler alchimique, mais ce type de four est également utilisé par les alchimistes de la fin du Moyen Âge.

La distillation au Moyen Âge permettait de produire de l'alcool à $60^{\circ}$ (aqua ardens) ou à $90^{\circ}$ (aqua vitae), de l'acide nitrique, utile pour séparer or et argent, de l'essence de roses pour les parfums, ou encore de l'essence de plantes diverses à des fins médicales. La distillation est donc à la base de l'industrie chimique naissante; aux $\mathrm{XV}^{\mathrm{e}}$ et $\mathrm{XVI}^{\mathrm{e}}$ siècles, les procédés se perfectionnent, surtout le refroidissement. Léonard de Vinci, par exemple, fera de la distillation et essaiera de perfectionner les appareils. Les ouvrages sur la distillation se multiplient à partir $\mathrm{du} \mathrm{Xv}^{\mathrm{e}}$ siècle, mais celle-ci constitue aussi l'une des phases des opérations alchimiques devant conduire à la réalisation du « grand-œuvre ».

Les sources iconographiques sont rares avant la Renaissance, même si les traités écrits au Moyen Âge sont nombreux. Cependant, à partir des années 1480, les manuscrits ornés de miniatures se multiplient. Avec l'imprimerie, les gravures sur bois font leur apparition dans les ouvrages, tout en ne détrônant pas la miniature que l'on retrouvera encore au $\mathrm{XVII}^{\mathrm{e}}$ siècle. Certaines planches de ces ouvrages représentent des fours proches de celui que l'on trouve au château de Meinsberg ${ }^{4}$.

4. À titre d'illustration, on peut citer des œuvres comme celles de Hiëronymus Brunschwygt, Liber de arte distillandi (Strasbourg, 1500), de Georgius Agricola, De re metallica (édité pour la première fois à Bâle en 1555), ou encore de Michael Maier, Tripus aureus (Francfort, 1618). 
Mais revenons justement à notre légende. Les romantiques, attirés par les sciences occultes, se firent le relais de la tradition alchimique, leur quête spirituelle et philosophique, jusqu'àl'ésotérisme etl'occultisme, supplantant alors la recherche de la transmutation du plomb en or. Goethe (1749-1832) fut l'un des premiers à s'intéresser à l'alchimie, par l'intermédiaire des écrits du théosophe illuminé Jakob Böhme (1575-1624); il s'essaya aux opérations de laboratoire et considérait que l'alchimie était une voie de connaissance, préférable à la connaissance officielle et universitaire. Cette conception se retrouve dans l'histoire de Faust, savant ou alchimiste, pactisant avec le diable. La première version de cette œuvre est publiée en 1808, puis en 1832.

$\mathrm{Du}$ côté français, on sait que Gérard de Nerval (1808-1855), profondément influencé par l'ésotérisme, traduisit le Faust de Goethe (la première édition date de 1828) et écrivit en 1831 un drame sur Nicolas Flamel avant L'Alchimiste en 1838, qu'il conçut avec Alexandre Dumas puis Les Illuminés, dans lequel apparait le mystérieux Cagliostro. Victor Hugo (1802-1885) enfin, dans Notre-Dame de Paris (1831), décrit le laboratoire de l'alchimiste Frollo, situé dans une des tours de la cathédrale. Influencé par les écrits du théosophe alchimiste Esprit Gobineau de Montluisant $^{5}$ (c. 1640-1658), il fait de la cathédrale et de ses sculptures une œuvre inspirée par le symbolisme alchimique.

C'est dans ce contexte littéraire des mêmes années - les érudits de la revue L'Austrasie ne pouvaient donc pas l'ignorer - que notre légende a été rapportée. Son origine historique restera toutefois obscure malgré ces rapprochements, tout comme la réelle utilisation du grand four du château.

Qu'en est-il enfin de la tour des Sorcières? Celle-ci était la tour la plus petite et sans doute la moins utilisable. Elle a donc été la première à être abandonnée. Au XVIII ${ }^{\mathrm{e}}$ siècle, le nom de cette tour était «Kesstour» ou « Keptour »; l'actuel nom, « Hexenturm » ou tour des Sorcières, a été adopté au XIX ${ }^{\mathrm{e}}$ siècle et ferait allusion à son état de délabrement précoce. Ayant en effet perdu sa toiture dès le milieu du XVIII ${ }^{\mathrm{e}}$ siècle, la tour ne pouvait donc plus abriter que des sorcières!

Le château, siège de la justice seigneuriale, devait cependant posséder une cellule. Les archives mentionnent qu'en 1558, des témoins rapportèrent le cas de l'emprisonnement au château d'un valet dénommé Thiellen Hanss de Niederleuken bientôt rejoint par sa mère, Thiellen Marich. Accusée de divers méfaits que les sources ne précisent pas, la mère du valet fut condamnée au bûcher et brûlée vive à Orscholz. Si cette anecdote était connue au XIX ${ }^{e}$ siècle, ce qui n'est pas improbable, le nom de la tour, adopté à cette époque, pourrait peut-être rappeler l'histoire de Thiellen Marich.

5. Voir l'Explication très curieuse des énigmes et figures hiéroglyphiques, physiques, qui sont au grand portail de l'église cathédrale et Métropolitaine de Notre-Dame de Paris, 1754. 


\section{Un château dans les brumes}

Pour terminer, je me permets de vous livrer un souvenir personnel, celui de ma première rencontre avec John Howe. Désireux de l'inviter à préparer une exposition au château de Malbrouck, projet qui n'a pu se réaliser à ce jour, j'avais rassemblé une série de photographies du château pour le convaincre de l'intérêt du lieu. Quand il regarda une photographie du château prise au loin, émergeant de la brume, John Howe me demanda si ce cliché avait été retouché, tant cette vue, vraiment « magique », l'avait séduit. Telle est bien la vue du château que l'on souhaiterait conserver en mémoire : à la fois celle d'un Moyen Âge réel et irréel.

Pourquoi cette image du château retient-elle immédiatement notre attention? Est-ce utile d'ailleurs de poser cette question. Au fond de nous même, nous connaissons déjà la réponse : cette image devient archétypale et synthétise le Moyen Âge ou tout au moins l'image que nous pouvons nous en faire, nourrie de nos lectures de jeunesse, de nos jeux de chevaliers et de princesse, de cinéma, comme de la présence de nombreux vestiges médiévaux, mélangés peut-être à d'autres images d'un Moyen Âge recomposé au XIX ${ }^{e}$ siècle, telles que l'architecture et la littérature nous les livrent.

Ainsi ce château est un maillon de cette longue chaîne qui nous conduit à travers les siècles et qui demeure en nous comme un élément déterminant de notre culture occidentale. Moyen Âge archéologique, Moyen Âge historique, Moyen Âge recomposé, réutilisé, réimaginé, voire rejeté, il reste toujours très présent dans notre univers européen et sous des formes parfois surprenantes. Si les civilisations meurent, si les nations disparaissent et si les sociétés changent, le Moyen Âge reste encore aujourd'hui un socle commun et une source d'inspiration.

J'espère que ce rapide voyage, ces allers et retours entre histoire et légende, ruine et restauration, imaginaire romantique et archéologie, bref entre le château et sa représentation, vous aura convaincus que ce lieu est le meilleur qui soit pour réfléchir sur le médiévalisme, sur la modernité du Moyen Âge.

Éric Necker Conservateur en chef du Patrimoine-Département de la Moselle 